Int. J. Dev. Biol. 61: 17-27 (2017)

doi: $10.1387 / \mathrm{ijdb} .160370 \mathrm{~mm}$

\title{
Models of amphibian myogenesis - the case of Bombina variegata
}

\author{
LEOKADIA KIEŁBÓWNA and MARTA MIGOCKA-PATRZAŁEK* \\ Department of Animal Developmental Biology, Institute of Experimental Biology, University of Wroclaw,
} Wroclaw, Poland

\begin{abstract}
Several different models of myogenesis describing early stages of amphibian paraxial myotomal myogenesis are known. Myoblasts of Xenopus laevis and Hymenochirus boettgerichange their position from perpendicular to parallel, in relation to axial organs, and differentiate into mononucleate myotubes. In Bombina variegate the myotomal myoblasts change their shape from round to elongate and then differentiate into mononuclear, morphologically mature myotubes. In Pelobates fuscus and Triturus vulgaris, myoblasts fuse into multinuclear myotubes. Mono- and multinucleate myotubes achieve morphological maturity during the differentiation process. During myogenesis of $B$. variegata, the nuclei of mononucleate, differentiating myotubes contain a tetraploid quantity of DNA (4C DNA). The stable quantity of DNA is confirmed by lack of ${ }^{3} \mathrm{H}$-thimidine incorporation into myotube nuclei. This outcome is a proof that myoblasts withdraw from the cell cycle in the $\mathbf{G 2}$ phase. The further development of myotomal myotubes involves myoblasts of mesenchymal origin. These myoblasts fuse with myotubes in X. laevis and $B$. variegate in the $\mathrm{G} 1$ phase. Secondary muscle fibres in amphibian myotomes have only mesenchymal origin. Mesenchymal myoblasts fuse into multinucleated myotubes. Myofibril development in the differentiating myotube and lack of DNA replication confirm the classical paradigm of myogenesis. Mesenchymal myoblasts are taking part in the myogenesis of musculus rectus abdominis and limb muscles. The mesenchymal cells in the myogenesis process show one model of myogenesis, which is a classical model of myogenesis. The mesenchymal cells probably come from dermatome.
\end{abstract}

KEY WORDS: myogenesis, myoblast, myotomal myogenesis

\section{Introduction}

Pre-somitic mesoderm (PSM) is the source of vertebrate skeletal muscles. Its metamerisation leads to formation of numerous, bilaterally and symmetrically arranged pairs of somites. In chicken, the somite is formed by loosely arranged cells of segmental mesodermal plate, which elongate and then adopt a radial arrangement. The somite is vesicle-shaped, with its lumen - myocoel - filled with cells in its centre (Bellairs, 1979).

Somitogenesis is an autonomous process. Internal properties of PSM cells in the somite formation are based on models of internal oscillator or clock (Dale and Pourquié, 2000). Cook and Zeman (1976) proposed the model of "clock and wavefront". The discovery of cyclic expression of mRNA of gene $c$-hairy1, transcription factor bHLH, in chicken PSM cells, was the first indicator of the oscillatory molecular clock which functions in PSM cells, before the somites appear (Palmeirim et al., 1997). The discovery of periodic expres- sion of genes Her1 and DeltaC in fish PSM cells suggests that the segmentation clock is conservative and is present in all vertebrates (Sawada et al., 2000). During one oscillation cycle, expression of mRNA of gene hes 1 passes as a caudo-rostral wave through PSM cells at cyclic time intervals during which consecutive somites are cut off (Jouve et al., 2000).

The embryologist's interest in somite development in Anamniota is small. Exceptions are Anura: Xenopus laevis (Keller, 2000), and Teleostei: Brachydanio rerio (rev. in Stickney et al., 2000). In amphibians the somite develops into centrally located myotome,

\footnotetext{
Abbreviations used in this paper: Amphi MDF, amphioxus myogenic determinant factor; bHLH, basic helix loop helix; eFGF, embryonic fibroblastic growth factor; FGF, fibroblast growth factor; MRF, muscle regulatory factor; NCAM, neural cell adhesion molecule; PCNA, proliferating cell nuclear antygen; PSM, pre-somitic mesoderm; SEM, scanning electron microscope; TEM, transmission electron microscopy; TGF $\beta$, transforming growth factor $\beta$.
}

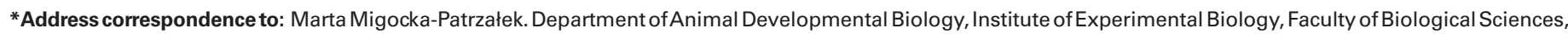
University of Wroclaw, Sienkiewicza 21, 50-335 Wroclaw, Poland. e-mail: marta.migocka-patrzalek@ uwr.edu.pl - web: www.zbrz.uni.wroc.pl (iD) http://orcid.org/0000-0003-0077-7779
} 
dorso-lateral dermatome and ventro-medial sclerotome (Malacinski et al., 1989; Radice and Malacinski, 1989; Wilson et al., 1989).

Anamniota somites are formed much like those of Amniota, as a result of PSM segmentation. In amphibians segmentation of paraxial mesoderm is highly conservative. However, as opposed to other vertebrates, in Anura, a representative of the family Pipidae, Xenopus laevis, paraxial mesoderm is initially two-layered, with a coelom between the layers. The coelom disappears when the myotome and dermatome are formed. The pre-myotomal cells are initially oriented perpendicular to the long body axis and then rotate by $90^{\circ}$.

A common character of vertebrates is the epithelial structure of their somites, which are rosette shaped in cross section. In amphibians, Urodela, for example in the axolotl, the myocoel, as opposed to Amniota, does not contain mesenchymal cells in its centre (Hamilton, 1969). Exceptions are Anura, in which the rosette does not occur.

The anamniote myotome is the largest part of the somite, which develops in situ. The sclerotome development in Amphibia is poorly known (Scaal and Wiegreffe, 2006). In Xenopus, at the late tail bud stage, only a small number of polymorphic cells was identified; the cells were situated on the ventro-medial margin of the somite, and had probably developed from a few myotomal cells (Malacinski et al., 1989; Wilson et al., 1989). The dermatome in Xenopus laevis is not segmented. It is a layer of cuboid cells which in later development covers the myotome's lateral surface (Hamilton, 1969; Keller, 2000; Youn and Malacinski, 1981a). The molecular mechanism of formation of the somite subdomains in Anamniota is unknown (Scaal and Wiegreffe, 2006). The studies of the last decade resulted in an original suggestion. It is suspected that the dernatome of Xenopus laevis has many characteristic features of the amniote dermomyotome (Grimaldi et al., 2004).

In the history of myogenesis studies, in vitro research on proliferating cells of forming pectoral and limb muscles of chicken/quail is crucial. Such studies showed that, having completed proliferation, the cells irreversibly exited the cell cycle in phase G1. The post-mitotic myoblasts fuse into multinucleate myotubes (Holtzer, 1970). The myoblasts at different phases of the cell cycle: S, G2 and during mitosis, are incapable of fusion (Bischoff and Holtzer, 1969; Holtzer, 1970; Okazaki and Holtzer, 1966). Cytophotometric DNA studies revealed that the myotube nuclei contained diploid quantities of DNA (Strehler et al., 1963). The myotube nuclei do not synthesise DNA, and thus can not divide mitotically or amitotically (Bischoff and Holtzer, 1969; Stockdale and Holtzer, 1961; Stockdale and O'Neill, 1972). Synthesis of myofibril proteins is associated with the cell cycle. Autoradiographic studies with ${ }^{3} \mathrm{H}$-thimidine and immunocytochemical studies in order to detect myosin and tropomyosin showed that microfilaments displayed positive reaction only in phase G1 (Bischoff and Holtzer, 1969; Okazaki and Holtzer, 1966). The basic paradigm implies that proliferation and morphological differentiation are mutually exclusive. DNA replication and synthesis of myofibril proteins exclude each other (Stockdale and O'Neill, 1972). The cell membranes disappear during the myoblast fusion (Lipton and Konigsberg, 1972). The myotube growth is affected by those myoblasts, which have not fused at the initial stage of fusion and thus can enter a new cell cycle. Having exited the cycle in phase G1, they fuse with the myotube or form new myotubes (Holtzer, 1970). The presented process follows the classical model.
The process of myoblast-myoblast fusion is at present extensively studied at molecular and genetic levels (rev. in Kiełbówna and Migocka-Patrzałek, 2014). The fusion is preceded by changes in the cell shape: elongation, smoothing of their surface, followed by adhesion and fusion. Transcription factors, which govern the morphological changes of the myoblasts, are unknown. The mechanism of the myoblast fusion will be understood when the transcription factors become known; these must be coordinated with morphological changes of the myoblasts (Krauss, 2010).

The mechanism of myoblast-myotube fusion is poorly known. Physico-chemical research of Orlov et al., (1989) shows that arising contacts between the cell membranes lead to membrane invagination, formation of free vesicles and to pinocytosis. The process leads to membrane interruption and loss of membrane fragments. Passing from invagination to vesicles depends on the osmotic gradient of the fusing cells. The gradient is determined by polymerisation of myofibril proteins in one of the fusing cells. The process takes place in the myotube (Orlov et al., 1989).

The key factors in arising of the skeletal muscle phenotype are four genes coding for MRFs (Muscle Regulatory Factors) proteins: MyoD, Myf5, myogenin and Myf4, of bHLH type (basic Helix Loop Helix) (Weintraub et al., 1991; Berkes and Tapscott, 2005). These proteins initiate expression of muscle-specific genes through binding with the control element of DNA, E-box or the promotor region of the target genes (Olson, 1990). Heterodimers, members of the MRFs with commonly occurring E2A proteins facilitate the process (Olson and Klein, 1994).

Early development of vertebrates is characterised by the presence of transcription factors Pax3 and Pax7. Pax3/Pax7-positive cells originate from the dermomyotome where their genes are already active (Bajard et al., 2006; Buckingham, 2006).

The expression of the gene coding for the transcription factor Lbx1 is limited to the migrating future muscle cells (Jagla et al., 1995). It is suggested that gene $L b x 1$ determines the myogenic properties of the migrating cells and is important for the recognition of signals which direct the cells and maintain their migrating potential (Brohmann et al., 2000; Christ and Brand-Saberi, 2002). The molecular nature of these signals is studied at present.

Early stages of chordate myotomal myogenesis show its great diversity. This diversity provides valuably material for comparative studies. In Tunicata the mononucleate transversely striated cells arrange in two bilaterally symmetrical bands running the length of the tail. Tunicata tail muscle shows no metameric structure. In Halocynthia roretzipresumptive muscle cells of the B-lineage have extensive potential for self-differentiation under the influence of material cytoplasmic determinants (Nishida and Sawada, 2001). Amphioxus is a key organism for understanding evolution of complex vertebrate myotomal myogenesis. The large, main compartment of each somite is the myotom. All of the myotomal cells differentiate synchronously and becoming striated muscle cells constituting the segmental blocks. The myotome is composed of more than one thousand thin lamellae ( $1 \mu \mathrm{m}$ thick). Throughout life each myotome lamellae remains monocucleate. Brianchiostoma belcherihas only one myogenic gene Amphi MDF (Amphioxus Myogenic Determinant Factor) in its genome; it codes for the bHLH protein only. The amino acid sequences of the bHLH domain and its phylogenetic analysis indicate great similarity of the bHLH domain and MyoD in vertebrates. It has been suggested that the gen Amphi MDF is not only a sister gene but also the ancestor of the four myogenic 
genes of vertebrates, therefor its duplication may be the origin of the MyoD gene family in vertebrates (Yuan et a., 2003). Myogenesis is poorly known in lamprey and hagfish. In lamprey larvae (amocoetes), similar as in Amphioxus myotomes, is composed of lamellae stacked horizontally. Each lamella has one nucleus. In adult lamprey the myotome is built of slightly flattened multinucleate muscle fibres (Flood et al., 1977). Myotomal muscle fibres of dogfish are nearly cylindrical rather than flat (Rovainen 1979). In "ancient" teleost such as Acipenser stellatus the myotome is built of wedgeshaped, flattened multinuclear lamellae. Most of the lamellae are transformed into irregular cylindrical fibres (Flood et al., 1987). In Brachydanio rerio myogenesis starts in adaxial cells. Adaxial cells migrate onto lateral surface of the embryonic myotome. Following migration they differentiate into mononucleate slow-twitch muscle fibres. Non-migrating cells of the embryonic myotome differentiate into fast-twitch muscle fibres. Myotomal cells become polynuclear through fusion (Devoto et al., 1996).

\section{Different models of myotomal myogenesis in Anura and Urodela}

\section{Myotomal myogenesis in Xenopus laevis (Pipidae) Anura}

\section{MyoD and Myf-5 in early development}

In the development of Xenopus, accumulation of MyoDtrancripts in the cells of paraxial mesoderm at the medium gastrula stage is the molecular response to the inducing factors FGF and TGF $\beta$ which are emitted from the newly formed endoderm (Hopwood et al., 1989). However those cells while expressing of MyoD do not realize complete differentiation programme (Buckingham, 2003).

The temporary release of mesodermal cells from mutual contacts at the stage of early and medium gastrula, studied in vitro, inhibits differentiation. Formation of a large group of cells is the prerequisite to entering the myogenesis phase. These observations provide grounds for an original hypothesis, which assumes that differentiation requires cell-cell interaction. Such interaction is called "communication effect". In the "communication effect" expression of muscle-specific transcription factors of MyoD in Xenopus requires around 100 cells to remain in contact (Gurdon et al., 1993; Buckingham, 2003). It was found that eFGF (embryonic Fibroblastic Growth Factor) participated in the cell contact; it is probably an endogenous factor in the communication (Standley et al., 2001). Other factors are cadherins, which participate in the signal communication between the cells and are necessary for the activation of MyoD (Holt et al., 1994). At the late gastrula and neurula stages, the cells lose their communication dependence and can differentiate without their original neighbourhood (Gurdon et al., 1993; Hopwood et al., 1992). Parallel with the effect MyoD, Myf-5 participates in myogenesis initiation. Combined action of MyoD and Myf-5 genes emphasizes their simultaneous function as myogenesis promoters (Hopwood et al., 1992).

The level of MyoD expression at the late gastrula stage in Xenopus is similar in the cells of pre-somitic mesoderm in both its anterior and posterior parts. However, at the initial stage of somitogenesis, strong expression of MyoD occurs in the nuclei of cells of the newly formed somites in the posterior part, and is absent from the cells of older somites in the anterior part. The fact suggests that MyoD, after initiation of myogenesis in Xenopus, is gradually degraded. The high level of MyoD expression is required for the initiation of myogenesis but not for maintaining it (Harvey, 1992).

\section{Paraxial mesoderm segmentation}

Paraxial mesoderm segmentation in amphibians is highly conservative. In Xenopus somitogenesis starts at the stage of late neural folds and continues after hatching (Blackshaw and Warner, 1976). As opposed to other vertebrates, only in the Anuran family Pipidae: Xenopus laevis and Hymenochirus boettgeri, the cells change their shape and position parallel to somitogenesis. They change their orientation from medio-lateral to cranio-caudal, rotating by $90^{\circ}$ (Hamilton, 1969; Kiełbówna, 1980; Youn and Malacinski, 1981a; Youn and Malacinski, 1981b; Daczewska, 2001;). After the rotation has been completed, the cell length corresponds to the somite length. In Xenopus the somite width corresponds to 10 cells (Hamilton, 1969). Around 150 cells take part in the rotation (Blackshaw and Warner, 1976) (Fig.1A).

Observations in scanning electron microscope (SEM) showed that individual rotating cells were responsible for the change of position of the whole somite (Youn and Malacinski, 1981a). This was confirmed by three-dimensional morphological visualisation of the rotating cells. Analysis in confocal microscope showed that during somitogenesis the cells elongated medio-laterally in a coordinated way, and then diverged toward the newly formed somite. Filopodia formed at their distal ends. The number of filopodia increased with progressing reorientation of the cells. The change in shape and orientation of each cell in the somite led to rotation of the whole somite by $90^{\circ}$. The somites were formed at regular intervals, in Xenopus of approximately every $50 \mathrm{~min}$. The molecular mechanism of the process is unknown (Afonin et al., 2006).

Gene Mespo is expressed in the cells of pre-somitic mesoderm (PSM) of Xenopus laevis (Joseph and Cassetta, 1999). Mespo codes for a protein which is highly homologous to the protein of the transcription factor subfamily bHLH and 54-60\% identical with the bHLH domain of protein Thylacin 1 which takes part in the segmentation (Sparrow et al., 1998). The expression of Mespo gene starts in the embryo's pre-somitic mesoderm, at the gastrula stage, and continues through the tail bud stage when it is especially strongly marked and when somitogenesis starts (Joseph and Cassetta, 1999).

The segmentation includes two phases. The first involves organising non-segmented mesoderm into groups of cells called somitomeres, the second consists in transformation of the somitomeres into somites. In Xenopus laevis the cells of somitomeres form somites through separation of a group of cells from the non-segmented mesoderm and changes of their orientation by $90^{\circ}$ in relation to the embryo's long axis. Based on the expression pattern of Thylacin 1 it was found that Thylacin 1 took part in delimiting the boundaries between the somitomeres. The expression of gene Thylacine1 is part of the complete signal pathway Notch (Sparrow et al., 1998). The genes, which code for the components of the Notch signal pathway are required for the division of paraxial mesoderm into repeated segmental structures, somitomeres. The Notch pathway plays the key role in both formation and maintenance of segmental identity of the somitomeres (Jen et al., 1999).

\section{Mononucleate myotubes in myotomal myogenesis}

In the myotomal myogenesis of Xenopus laevis (Pipidae) it was found for the first time that myoblasts developed directly into mononucleate myotubes (Fig.1A). Their length was at the 
same time the myotome length. A single large nucleus is centrally located in myotubes. The number of mature myofibrils increased in the differentiating mononucleate myotubes (Kiełbówna, 1966).

Analysis performed using TEM indicate that myofibrilogenesis starts very early in the cells of the forming somites. Thin and thick filaments in the form of bundles organise into sarcomeres with $Z$ line and bands $A$ and I. At the same time sarcotubular system is formed. Cells in the cephalic somites at stage 24 for the first time contract spontaneously or upon stimulation. Mononucleate myotubes obtain morphological and functional maturity during their differentiation (Blackshaw and Warner, 1976; Muntz, 1975).

In the differentiating mononucleate myotube at stages 26-42, the quantity of nuclear RNA, examined cytophotometrically, increases in the nucleoplasm and in the nucleoli. The increase in the quantity of nuclear RNA indicates an increased transcription expression of the corresponding genome segments. At the same time the size and basophily of the nucleoli increase. A strongly basophilic component appears in the originally small and poorly staining nucleolus. According to (Sameshima and Shiokawa, 1969) the poorly and strongly staining components in the nucleoli of embryonic cells of Xenopus laevis in TEM correspond to the fibrillar and granular parts of the nucleolus. Then a poorly staining vacuole appears within the strongly basophilic component of the nucleolus. The growth of nucleoli, accompanied by the appearance of the strongly basophilic component and the vacuole, testifies also to the expression of nucleoli in the differentiating mononucleate myotube (Kiełbówna, 1973).

The early amphibian development takes place in a "closed system". The substances required for the development derive from yolk platelets (Brown, 1964) and are gradually released during vitellolysis. It was found that in Rana temporaria and Bufo bufo utilisation of the material from yolk platelets and lipid droplets did not start at early development stages, for example blastula (Romek, 1998). Yolk platelets and lipid body were cytochemically examined in mononucleate myotubes of Xenopus laevis at stages 32-42. The results of safranine and fast green staining are compatible with the platelet structure in TEM. The crystalline core of the platelet stains with safranine, the granular superficial component stains with fast green (Selman and Pawsey, 1965). During vitellolysis the thin fast green-positive layer of the plate shows increasingly weaker staining. TEM examination showed that the layer released molecules of 40-60 $\AA$ (Karasaki, 1963). The safranine-staining core of the platelet changes into fast green-positive. The change is associated with the change of the core form from crystalline to granular. The platelet core is built of phosphoproteins and lipoproteins at molecular ratio of 2:1. The loss of affinity to safranine is also associated with dephosphorilation of phosphoproteins (Selman and Pawsey, 1965). Histones and non-histone proteins were cytochemically detected in yolk platelets. The yolk pletelets contain phospholipids. No polysaccharides and acid mucopolysaccharides were detected in the platelets. Likewise, specific cytochemical methods to detect nucleic acids: DNA and RNA, yielded negative results. The lack of positive reaction results from the very small DNA and RNA content in individual yolk platelets (Kiełbówna, 1975). The presence of DNA and RNA in yolk platelets of Xenopus laevis was detected biochemically, autoradiographically and in TEM (Hanocq-Quertier et al., 1968; Kelley et al., 1971).

\section{Mechanism of transformation of mononucleate myotubes into multinucleate fibres}

The process in Xenopus laevis has remained obscure till recently. Three papers report on different results. It was thought, for example, that the smaller nucleus in the myotube arose as a result of unequal, amitotic division of the myotube nucleus, which then underwent multiple mitotic divisions. Cytophotometric studies of Feulgen-positive nuclei showed that the nuclei of mononucleate myotubes (stage 35/36) approached tetraploidy. At stage 40, the myotube nuclei exceeded the tetraploid quantity and approached
A

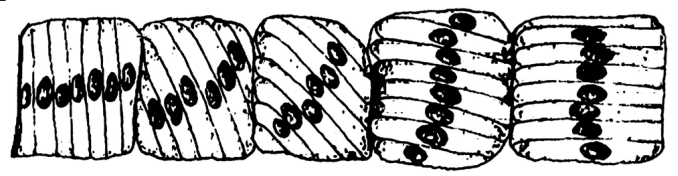

B

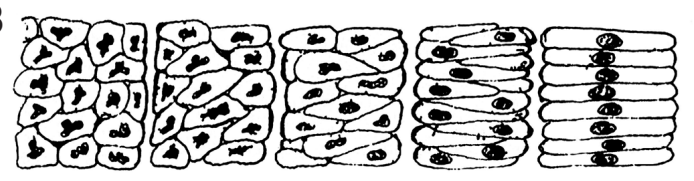

C

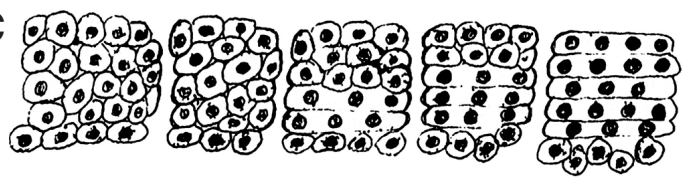

D

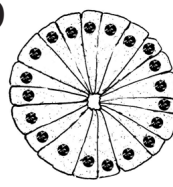

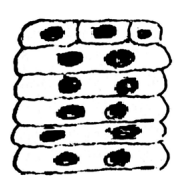

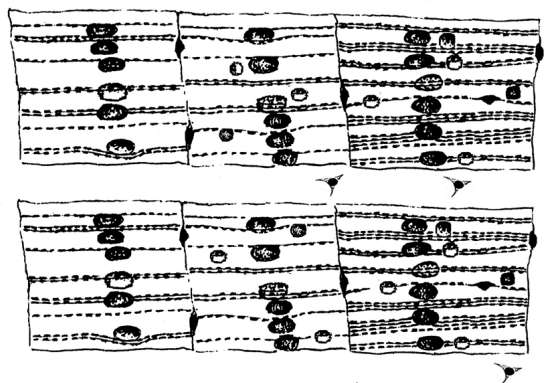

$+1$

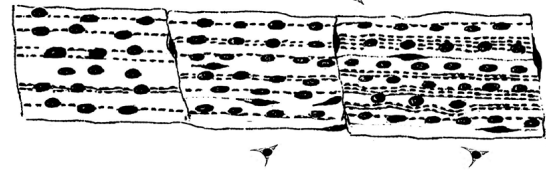

h.

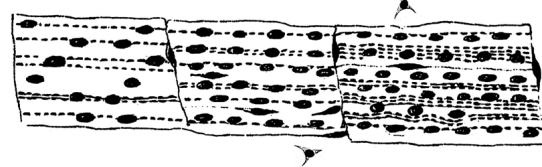

Fig. 1. Different models of myotomal myogenesis. Consecutive stages of muscle fibre differentiation from left to right. (A) Xenopus laevis. Cell rotation. Differentiation of mononucleate myotubes, in myotube several mature myofibrils. On myotube surface elongated mesenchymal cells. In myotube one primary large nucleus and smaller secondary, mesenchymal nuclei. (B) Bombina variegata. In somite compact groups of rounded cells. Myoblasts change shape, elongate to occupy the whole myotome length. Near myotubes mesenchymal cells present. Nuclei in myotube differ in size: primary nucleus is larger, secondary, mesenchymal nucleiare smaller. Transversely striated myofibrils. (C) Pelobates fuscus. In somite compact group of polymorphic rounded cells. Linearly arranged myoblasts fuse into myotubes of several nuclei. Myotubes differentiate, a few mature myofibrils. After mesenchymal cells have immigrated into myotomes, the number of nuclei in myotubes increases. More numerous mature myofibrils (Kietbówna, 1981). (D) Triturus vulgaris. Rosette-shaped somites. Fusing myoblasts most often form binucleate myotubes. Myotubes grow and differentiate, several striated myofibrils. Elongated mesenchymal cells appear on myotube surface. Number of nuclei in myotubes increases; length of differentiating myotubes increases as well, more numerous mature myofibrils (Daczewska \& Kietbówna, 2000). 
octoploidy. At stage 45, smaller nuclei appeared in the myotube. The larger, primary nuclei contained quantities of DNA approaching tetraploidy. The smaller nuclei formed one, diploid class. At stage 51 , the smaller nuclei belonged to the same diploid class (Kiełbówna, 1966).

In the multinucleate myotube of Xenopus laevis, the size of the larger, primary nucleus is $6 \times 18 \mu \mathrm{m}$, that of the smaller nuclei is $4 \mathrm{x}$ $10 \mu \mathrm{m}$ (Muntz, 1975). Cytophotometric studies of DNA of myotube nuclei were done on sections $5 \mu \mathrm{m}$ and $7 \mu \mathrm{m}$ thick. The myotube nuclei which did not contain full quantity of $4 C$ DNA were probably measured on thinner sections, of $5 \mu \mathrm{m}$. The nuclei which exceeded that quantity were probably measured on thicker sections, of $7 \mu \mathrm{m}$. The earlier observed mitotic figures in the myotube represented mesenchymal cells situated on the myotube.

Muntz (1975) suggested that the source of smaller nuclei in the myotube were the satellite cells, located (at stage 42) at the ends of the myotube and fusing with it. Primary myotubes originate from all the myotomal cells and are formed synchronously in each myotome. Thus they are not satellite cells. At stage 42 the cells at the ends of the myotube are mesenchymal cells.

The third suggestion, based on TEM studies, assumed that multinucleate myotubes were formed as a result of multiple amitotic divisions of the myotube nucleus. The proof would be the deep invagination of the nuclear envelope which would lead to the division of the nucleus (Boudjelida and Muntz, 1987). The TEM image may have registered a nucleus movement. In in vitro myogenesis studies it was found that the invagination of nuclear envelope, even reaching as deep as half nucleus diameter, occurred during the nucleus movement in the sarcoplasm (Cooper and Konigsberg, 1961).

Nicolas et al., (1998) think that the mechanism which is responsible for the formation of multinucleate muscle fibres in the myotomes of Xenopus laevis is the octoploid DNA quantity in the myotube nuclei and the amitotic nucleus division. However these authors cannot exclude that some secondary migrating myoblasts could take part in the multinucleation process.

The various suggestions regarding the mechanism of origin of multinucleate myotubes in the myotomes of Xenopus laevis required further studies. Autoradigraphy with ${ }^{3} \mathrm{H}$-thimidine, not used earlier, provided the explanation. The nuclei of mononucleate myotubes do not incorporate the labelled thimidin and thus can not divide mitotically or amitotically. The smaller nuclei, which appear in the myotube, are of mesenchymal origin (Fig 1A). The diploid quantity of DNA in the smaller nuclei, as demonstrated in the earlier studies, shows that the mesenchymal cells prior to the fusion exit the cell cycle in phase G1. TEM studies confirmed the fusion of the mesenchymal myoblasts with the myotube. Subsarcolemmal vesicles appear in the myotube cytoplasm at the stage of elongated mesenchymal cells adhering to the myotubes. Gaps which open the road to fusion of the myoblast sarcoplasm with the myotube appear in the cell membranes in the site of fusion (Kiełbówna and Daczewska, 2005). The appearance of vesicles in only one of the fusing cells, i.e. the myotube, and the loss of the fragment of myoblast and myotube membranes in the site of fusion, are compatible with the results of physico-chemical studies of Orlov et al., (1989) (see Introduction).

Secondary myotubes develop in the neighbourhood of the primary fibres in Xenopus laevis. They result from fusion of elongated myoblasts of mesenchymal origin. As opposed to the primary fibres, of mesodermal-mesenchymal origin, the secondary fibres are of mesenchymal origin (Daczewska, 2006).

A forming myofibers in Xenopus laevis can have different destinies. It is established that the primary myotomal myofibers, which die, are progressively replaced by secondary multinucleated myofibers arising from fusion of recently migrated adult-type myoblasts during metamorphosis (Nishikawa and Hayashi, 1994; Chanoine and Hardy, 2003).

\section{Myotomal myogenesis in Hymenochirus boettgeri (Pipidae) Anura}

In somitogenesis of Hymenochirus boettgeri blocks of cells, somites, split off the non-segmented mesoderm at stages 20-23. The somitogenesis is accompanied by cell rotation in which the cells change their orientation from perpendicular to parallel in relation to the axial organs. The change of orientation starts in the dorsal part of the somite and progresses ventralward. TEM studies showed that the plasmalemma of the somite cells formed numerous long and thin processes in the terminal parts of the cells and shorter processes on their lateral surfaces. These structures are probably engaged in movements of the cells relative to each other in the process of rotation. The first myofilaments, arranged in bundles with strongly marked electron dense line Z, appear in the myoblasts at stages 20-23. Immunocytochemical studies showed a positive reaction of MyoD in the nuclei of segmented mesoderm myoblasts, the myoblasts during rotation and in the myoblasts, which occupy the whole length of the myotome. Skeletal $\alpha$-actin was detected in the terminal parts of the myoblasts, which occupy the whole myotome length. At stages 30-35 the myoblasts differentiate into mononucleate myotubes. The process involves all the myoblasts in the myotome at the same time. Mature myofibrils are present in the myotubes. Yolk platelets undergo vitellolysis. At further stages (stage 37) mesenchymal cells appear in the intemyotomal gaps, and at stages 38-40 they become located in the myotomes, between the myotubes. Interestingly, transcription factor MyoD was detected in the nuclei of the mesenchymal cells, which adhered to the myotubes. Its presence testifies to the acquisition of myogenic properties by the mesenchymal cells prior to their fusion with the myotubes, perhaps under the effect of the myotube's induction activity. The mesenchymal cells which have remained in the intermyotomal gaps acquire fibroblastic properties. This is demonstrated by collagen fibres with appear in their neighbourhood.

Numerous subsarcolemmal vesicles appear in the myotube cytoplasm in the site of adhesion of the secondary myoblasts (of mesenchymal origin) to the myotubes. Their presence precedes the myoblast-myotube fusion. Also gaps which open the road to cell fusion arise in the adhering cell membranes (Daczewska, 2001).

Densitometric DNA studies in the myotomal myogenesis of $H$. boettgeri showed that the myotube nuclei at stage 35 reached the DNA quantity of $3 \mathrm{C}$, while at stage 42 the most numerous nuclei reached the quantity of $4 \mathrm{C}$ DNA. In the smaller nuclei, of mesenchymal origin, at stage 42, the DNA quantity corresponded to the diploid 2C DNA. Immunocytochemical studies on PCNA (Proliferating Cell Nuclear Antygen) indicate that at stages 35 and 42 the primary myotube nuclei react positively. At stage 42 positive reaction is also displayed by the smaller nuclei of mesenchymal origin. At stage 45, PCNA was not detected in the larger nuclei of the myotubes. Positive reaction was also observed in the nuclei of the mesenchymal cells located between the myotomes and in 
the myotome, between the myotubes. The presence of PCNA in the nuclei of the primary myoblasts (stage 42) is in agreement with the increasing quantity of DNA, which in those nuclei reaches the tetraploid level (4 C DNA). The absence of PCNA reaction in the primary nuclei of the myotubes (stage 45 ) suggests the exit of the cells from phase S. The presence of PCNA in the smaller nuclei of the myotube (stage 42) is unclear (Daczewska and Saczko, 2005).

\section{Myotomal myogenesis in Bombina variegata (Discglossidae) Anura}

Paraxial mesoderm of Bombina variegata, at the stage of neural plate, is built of compact cells with rounded shape. The first somites (stage 19) have a similar structure. Only as late as stage 23, the myoblasts change their shape from rounded to elongated. The process involves all the myotomal cells. As a result, the myoblasts achieve the whole mytome length. The elongated myoblasts differentiate into mononucleate myotubes (Fig. 1B). Light microscope studies showed that myofibrils with sarcomeres were formed in the differentiating mononucleate myotube. The number of myofibrils increases in growing and differentiating myotubes. At stage 37, mesenchymal cells appear in the intermyotomal gaps, and then migrate to the myotomes, between the myotubes. Smaller nuclei appear in the myotube (stage 45). They are nuclei of mesenchymal cells which have fused with the myotube (Fig. 1B).

Autoradiographic studies with ${ }^{3} \mathrm{H}$-thimidine showed that at stage 26, after 6 hours of incubation, nuclei of the cells of nonsegmented mesoderm were labelled with ${ }^{3} \mathrm{H}$-thimidine. After 18 hours of incubation also the myoblast nuclei in a few youngest myotomes which were formed during the incubation were labelled. In the closest older myotome the myoblast nuclei showed no traces of incorporating labelled thimidin. The nuclei of differentiating mononucleate myotubes did not incorporate ${ }^{3} \mathrm{H}$-thimidine. After formation of the multinucleate myotubes ${ }^{3} \mathrm{H}$-thimidine was not incorporated by the primary myotube nuclei and the nuclei of the secondary myoblasts of mesenchymal origin. Only nuclei of the mesenchymal cells which were located in the neighbourhood of the myotubes were strongly labelled.

Complementary cytophotometric studies on nuclear DNA showed that the nuclei of mononucleate myotubes (stage 28) in the youngest and the oldest myotomes formed one tetraploid class. At stage 41 , the myotube nuclei also belonged to one tetraploid class. In polykaryocytes (stage 52) the large nucleus remained in the tetraploid class, the small nuclei represented the diploid class (Fig. 2)

The absence of DNA replication and the tetraploid DNA quantity in the myotube nuclei show that the myotomal myoblasts of Bombina variegata have exited the cell cycle in phase G2. The results conform to the crucial regularity in myogenesis: DNA replication and myofilament synthesis are mutually exclusive. Myofibrils are formed in the myotubes which do not replicate DNA (Kiełbówna and Kościelski, 1979).

\section{Myotomal myogenesis inPelobates fuscus (Pelobatidae)Anura}

In the development of Pelobates fuscus, at the stage of advanced neural folds, small rounded cells of paraxial non-segmented mesoderm form a compact group. The first somites are similarly structured. At the stage of ten somites, in the oldest somites the myoblasts are arranged in a few rows along the myotome's long axis. Then the myoblasts of each row fuse into 4/5-nucleated myotubes. The myotube length corresponds to the myotome length. Myofibrils are
A

B
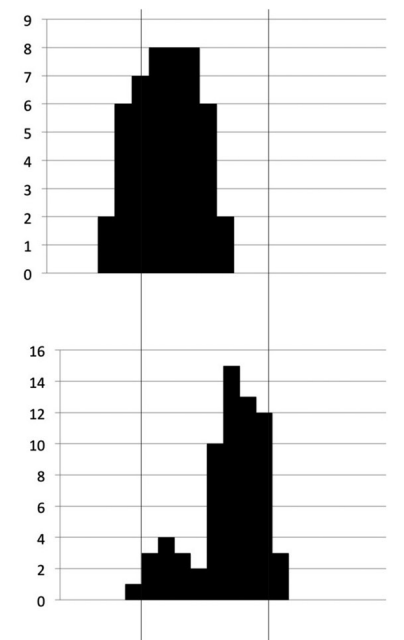

C

D

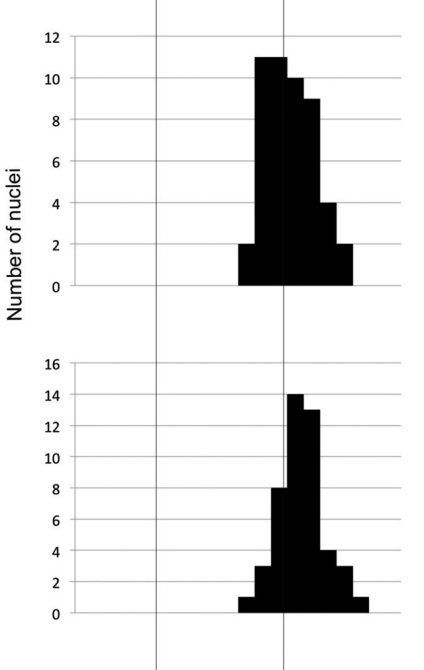

E

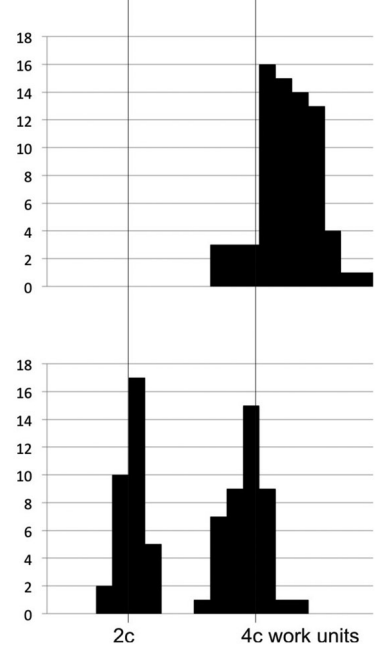

Fig. 2. Histograms of DNA content in nuclei at various stages of myotomal myogenesis Bombina variegate. (A) diploid DNA content in erythrocyte nuclei (control). (B) DNA in cells of non-segmented mesoderm. Two classes of nuclei: diploid and tetraploid. (C) tetraploid DNA content in youngest myotomal myoblasts at stage 28. (D) tetraploid DNA content in paracephalic myotomal myotubes at stage 28. (E) DNA in mononucleate myotubes of swimming tadpoles, stage 41. (F) DNA in polykaryocytes. Primary nuclei in tetraploid class, secondary nuclei of mesenchymal origin in diploid class (Kietbówna and Kościelski, 1979). 
formed in the growing and differentiating multinucleate myotubes (Fig. 1C). Yolk plates undergo vitellolysis. In the advanced stage of myotube differentiation, in the presence of the same number of nuclei in the myotube, myofibrils fill the myotube sarcoplasm.

In tadpoles $12 \mathrm{~mm}$ long, mesenchymal cells appear in the intermyotomal gaps and then migrate into the myotomes, between the myotubes. Numerous mitotic figures are observed in the mesenchymal cells. Elongated bipolar mesenchymal cells adhere to the myotubes and then fuse with them. The number of nuclei in the myotube increases to 10 . Following the fusion the new nuclei are usually located under the sarcolemma surface.

Analysis of cross sections through the myotomes showed that the medial part of the myotome was occupied by muscle fibres of a larger cross section area, with their sarcoplasm evenly filled with myofibrils. The fibres in the lateral part of the myotome had a smaller cross section area and, besides, a part of their sacroplasm was myofibril-free. Numerous granules in the myofibril-free sarcoplasm, observed in light microscope, may be mitochondria.

In tadpoles $18 \mathrm{~mm}$ long the number of nuclei in the medial fibres increases to 21. Cytoplasmic buds with nuclei appear on the lateral surface of the lateral fibres. The buds result probably from active growth of the perinuclear cytoplasm around the nuclei which were originally located under the sarcolemma. The presence of the buds is short-lasting.

The elongated bipolar mesenchymal cells form a row on the muscle fibre surface. Their fusion leads to formation of secondary myotubes. In tadpoles at advanced stage (length $28 \mathrm{~mm}$ ) all the medial and lateral fibres are accompanied by the secondary myotubes whose number increases in subsequent development stages. The difference in the cross section area between the primary medial and lateral fibres increases. In the last studied development stage the primary medial fibres have their cross section area five times larger than the lateral fibres. Interestingly, the secondary fibres, which develop in contact with the primary medial and lateral fibres become morphologically similar to their templates. In tadpoles of Pelobates fuscus $40 \mathrm{~mm}$ long terminal parts of muscle fibres form muscle-tendon junctions in the myosepts (Kiełbówna, 1987).

In Xenopus laevis two different types of fibres occupy two different zones in the somite. The thinner fibres occupy superficial positions while the thicker fibres form the core part of the somite. SEM studies showed that myofibrils filled a large part of the sarcoplasm of the thicker fibres, and the remaining marginal part was mitochondria-rich. In the thin fibres mitochondria occurred not only in the peripheral part but also between the myofibrils. The thicker fibres in Xenopus are white fibres while the thin superficial fibres are red fibres (Kordylewski et al., 1989). In the development of myotomal muscles in Pelobates fuscusthe morphologically different medial and lateral fibres correspond to the medial and superficial fibres in Xenopus laevis.

A myogenesis model similar to that described for Pelobates fuscus is also found in myotomal myogenesis of Bufo bufo, a representative of Bufonidae (Brustis, 1979) and in Rana lessonae, family Ranidae (Daczewska and Pałucka, 1999).

\section{Myotomal myogenesis in Triturus vulgaris (Salamandridae) Urodela}

The somite of Triturus vulgaris (stage 25-26) has a rosetteshaped cross section. Elongated cells of the monolayer wall of the rosette are directed toward the rosette's centre. The arising myotubes are most often bi-nucleate, probably resulting from fusion of the myoblasts located in the rosette's wall on the opposite side. Transversely striated myofibrils are formed in the differentiating myotubes. Yolk platelets undergo vitellolysis. With progressing differentiation (stages 33-35) the number of myofibrils increases. At stages $36-39$, in the caudal part the myotubes are mononucleate. This is probably a result of the smaller number of cells in the caudal somites. Mesenchymal cells migrate into the intermyotomal gaps; at stages 40-44 they are present in the myotomes, between the myotubes. Elongated, bipolar myoblasts of mesenchymal origin adhere to the myotubes and fuse with them. The number of nuclei in the myotubes increases (Fig. 1D). TEM studies showed that muscle fibres at stages 40-49 had a developed sarcotubular system, and sarcomeres with $\mathrm{Z}$ line were present in mature myofibrils. It was found that the nuclei of pre-fusion myoblasts, adhering to the muscle fibres, were heterochromatin-rich, and became euchromatic, genetically more active, following the fusion. Besides, large cytoplasmic vacuoles were observed in the cytoplasm of the pre-fusion myoblasts; the role of the vacuoles is unknown.

Collagen fibres were observed in the direct vicinity of the mesenchymal cells located in the myosept. The terminal parts of mature muscle fibres (stage 55) form muscle-tendon junctions (Daczewska and Kiełbówna, 2000).

In the myotomal myogenesis of the studied amphibians the role of mesodermal myoblasts is limited to the formation of mono- or multinucleate myotubes. The development of myotubes is synchronous. These myotubes are the founder myotubes. The mesenchymal myoblasts fuse with them. The mesenchymal cells will participate in the development of both primary and secondary myotubes. The origin of the mesenchymal cells, which take part in the amphibian myotomal myogenesis is still unclear. The mesenchymal cells are bipotential: myogenic and fibroblastogenic. Their myogenic function is testified to by their ability to fuse with the myotube and to form new myotubes. The appearance of collagen fibres in the direct neighbourhood of the mesenchymal cells in the myosept indicates their fibroblastic function. The hypothesis of Grimaldi et al., (2004) assumes that the dermatome of Xenopus laevis has many traits of amniote dermomyotome. The basis is the strong expression of $M y O D$ in the dorsal and ventral margins of the somite, associated with the dermatome lips. The cells of the amniote dermomyotome differentiate into myoblasts, fibroblasts and other cells. The bipotential mesenchymal cells in the amphibian myotomal myogenesis would, according to the hypothesis of Grimaldi et al., (2004), originate from the dermatome.

\section{Myogenesis of hypaxial muscles}

\section{Myogenesis of musculus rectus abdominis in Bombina bombina}

At stage 37, cells released from the neural crest migrate ventralward and, together with the sclerotome cells, join the released dermatome cells. At stage 39, these cells leave the somite. Having entered in contact with the somatopleura they cease to migrate. A temporary large accumulation of cells is formed. A similar structure was observed in the development of the muscle of ventral body wall in Xenopus laevis. The structure was described as "discrete patches clearly seperate from somites". MyoD expression was detected in its cells (Martin and Harland, 2001).

In Bombina bombina single cells separate from this structure 
and migrate ventralward. The development of the first muscle fibres starts at stage 40 .

Musculus rectus abdominis develops on the external surface of somatopleura. Histological analysis of myogenesis was performed on isolated somatopleura of a tadpole at stage 45. The development of muscle fibres on the surface of thin somatopleura resembles myogenesis studied in vitro. The youngest, long myotubes form as a result of fusion of bipolar myoblasts. Myotube differentiation starts very early. A single transversely striated myofibril was observed in a very thin myotube. Differentiating myotubes, in which the number of myofibrils is increasing are accompanied by numerous mesenchymal cells. Having changed their shape into spindle-like, these cells adhere to the myotubes and fuse with them. The increase in the number of nuclei in the myotube leads to the myotube growth; the number of mature myofibrils increases as well. The development of muscle fibres is asynchronous. New thin myotubes arise in the vicinity of the muscle fibres. Some young muscle fibres branch or anastomose, with fibroblast-like myoblasts taking part in these processes. In the forming anastomose the fibroblast-like cell occurs between two fibres. The process of the fibroblast-like cell fuses with the muscle fibre, another process of the same cell fuses with the other muscle fibre. Interestingly, the fibroblast-like cell takes part in differentiation. It was found that the transversely striated myofibril which was present in the first muscle fibre ran along the whole process of the fibroblast-like cell through the perinuclear cytoplasm to another process. The remaining processes of the cell did not participate in the anastomose formation.

Numerous melanocytes are present in the neighbourhood of muscle fibres. They originate from the neural crest. Cytophotometric studies on nuclear DNA of the muscle fibres showed that the muscle fibre nuclei formed one diploid class (2C DNA). The nuclei of the mesenchymal cells, which accompanied the muscle fibres formed two classes: diploid and tetraploid.

Collagen tendons develop in parallel with the development of muscle fibres. The tendons run from the myosepts ventralward. The muscle fibres between them run perpendicular to the tendons. The muscle-tendon junction of a young, thin fibre with the thin tendon does not resemble such junctions in mature muscle fibres. A knot-like thickening arises in the site of junction.

Five to six myomeres are present in the musculus rectus abdominis. Differentiation of muscle fibres in the myomeres shows a dorso-ventral gradient. Increasingly younger fibres are formed towards the ventral midline. At stage 50, the muscle fibres of all the myomeres are mature (Kiełbówna, 1993).

Analysis of total objects of Xenopus laevis showed that the cells destined for the development of ventral muscle occurred in the ventro-lateral margin of the somite and displayed increased regulation of Pax3. Pax3-positive cells migrated ventralward and underwent differentiation with expression of transcription factor Myf5, followed by expression of MyoD. Expression of genes NCAM (neural cell adhesion molecule) was also detected in the migrating cells (Martin and Harland, 2001). A new role of Lbx1 was discovered in early development stages of tadpoles of Xenopus laevis. As opposed to Amniota, expression of Lbx1 occurs in all the myoblasts, which participate in the development of the muscle of ventral body wall (Martin and Harland, 2006).

\section{Myogenesis of hind limb muscles in Xenopus laevis}

The limb bud (stage 48) is hemi-spherical. After one week it starts to elongate. The bud length exceeds its thickness (Muntz, 1975). No transcripts of regulatory factors (MRF) were detected in the limb bud till stage 50. After expression of Myf-5 and MyoD mRNA in limb bud (stage 50) myogenin mRNA strongly accumulated later at paddle stages (stage 52). Myogenin transcripts accumulated in a few small secondary myofibers (Nicolas et al., 1998).

The cells of the limb bud at stage 50 forms a loose matrix. The cells have large nuclei, a small quantity of perinuclear cytoplasm and numerous processes. Their cytoplasm and nucleoli are strongly basophilic. Then, two fields of accumulated mesenchymal cells form in the limb bud, on the dorsal and ventral side of the femur chondrous primordium. They are myogenic fields in which myogenesis starts. Numerous mitotic figures are observed in the mesenchymal cells of the myogenic fields. The cells located in the centre of the myogenic field cease to divide. They change their shape from polygonal to elongated, bipolar. Then they become linearly arranged and fuse into myotubes of several nuclei. The myotube cytoplasm and nuclei remain strongly basophilic. Several myotubes are formed at the same time. Transversely striated myofibrils appear in the myotubes. New myotubes are formed in

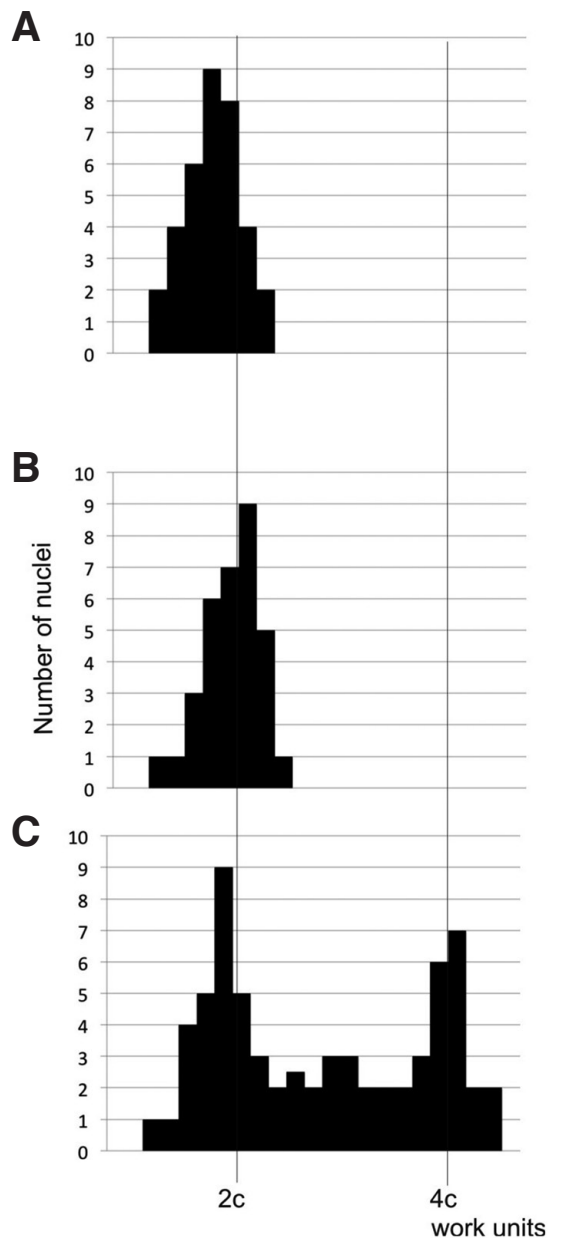

Fig. 3. Histograms of DNA content in nuclei of differentiating cells of limb muscle of Xenopus laevis. (A) diploid DNA content in nuclei of elongated myoblasts prior to fusion. (B) diploid class of nuclei in myotubes. (C) DNA in dividing mesenchymal cells. Diploid class of nuclei in post-mitotic phase, tetraploid class pre-mitotic (Kietbówna, 1980). 
the neighbourhood of the already existing ones. Older myotubes grow as a result of fusion with mesenchymal myoblasts. Basophily of the cytoplasm decreases in the growing myotubes. The intensity of nucleolus staining does not change. New mesenchymal cells join the myogenic field.

Cytophotometric studies on nuclear DNA showed that the DNA content in the nuclei of the elongated bipolar myoblasts was diploid (2C DNA) (Fig. 3A). The fact is important since it shows that the elongated bipolar myoblasts have exited the cell cycle and are in post-mitotic phase, G1. Diploid DNA content was also observed in the nuclei of mature, multinucleate myotubes which arose as a result of fusion of the elongated myoblasts and fusion of the myoblasts with the myotube (Fig. 3B). The mesenchymal cells in the myogenic field divided mitotically and formed two classes of nuclei: diploid and tetraploid (Fig. $3 C$ ). These results provided the basis for determining the diploid quantity of DNA (Kiełbówna, 1980). The myogenesis of the other limb muscles is most certainly similar.

In Elentherodactylus cogni (which has no tadpole stage) the development of limb muscles starts just after closing of neural tube; its starts with expression of Lbx 1 in the cells of the ventral part of trunk somites. Then the cells are observed at the base of developing limb bud. At later stages cells with Lbx1 expression are present among the mesenchymal cells in the limb bud (Sabo et al., 2009).

\section{Concluding remarks}

Analysis of early stage of myotomal myogenesis in Chordata indicates that myogenic process follows an evolutionary course. The process starts with myogenesis and lead to development of mononucleate cells witch either constitute final stage or represent a transitional form. Mononucleate muscle cells develop in tail of Tunicata. In Amphioxus the myotomal cells differentiate into mononucleate mature muscle lamellae (see introduction). Mononucleate myotubes, morphologically mature, develop in philogenetically old Amphibia, Xenopus laevis, Hymenochirus boettgeri, and Bombina variegate. They constitue the intermediate state in myogenesis. Their further development takes place on account of mesenchymal cells. Myoblast in another Anura, Pelobates fuscus, and Urodela, Triturus vulgaris, fuses into multinucleate myotubes.

The autonomic differentiation process can proceed in two different ways. In Tunicata presumptive muscle cells, the B-lineage (primary lineage), have extensive potential for self-differentiation under the influence of cytoplasmic determinantsø. In Xenopus laevis autonomous initiation of expression of $\mathrm{MyoD}$ in cells of non-segmented mesoderm (mid gastrula stage) is effect of communication comes from more than 100 cells. This "communication effect" taking place when MyoD expression occurs independently after mesodermal induction.

Myotomal myoblasts in Bombina variegata exit the cell cycle at phase G2. The basis is the tetraploid quantity of nuclear DNA (4C DNA). In the developing mononuclate myotube of Bombina variegata during myofibril formation there is no incorporation of $3 \mathrm{H}$-timidin in the nucleus. According to the basic paradigm of myogenesis, synthesis of myofilaments and DNA replication are mutually exclusive. The absence of DNA replication in the myotube nucleus of Bombina variegata and the myofibril development conform to the paradigm.

Myotube growth is associated with increase in the number of nuclei. Myotomal myotubes of Bombina variegata develop with one nucleus. According to Cheek et al., (1971) after Allen et al., (1999) in theory the myonuclear domain is a volume of cytoplasm supported by a single nucleus. In the myotube of Bombina variegata one tetraploid nucleus corresponds to two diploid nuclei. One tetraploid nucleus supports thus a double cytoplasmic domain. In such conditions the myotubes grow, differentiate and reach morphological maturity.

Amphibian dermatome is a thin monolayer structure. Assuming that the dermatome is the source of mesenchymal myoblasts in amphibian myogenesis, its cells should have a great developmental potential. The potential is manifest in their great mitotic activity.

Muscle fibres of mesenchymal origin, in both paraxial and hypaxial myogenesis, differentiate according to the same model. While myotomal myogenesis is much more varied among Amphibia, limb and abdominal myogenesis follow classic pattern.

\section{Acknowledgments}

Authors of this manuscript are supported by The Polish State Committee for Scientific Research, Projects No. 1068/S/IBE/2016.

\section{References}

AFONIN B, HO M, GUSTIN JK, MELOTY-KAPELLA C, DOMINGO CR (2006). Cell behaviors associated with somite segmentation and rotation in Xenopus laevis. Dev Dyn 235: 3268-3279.

ALLEN DL, ROY RR, REGGIE EDGERTON V (1999). Myonuclear domains in muscle adaptation and disease. Muscle and Nerve 22: 1350-1360.

BAJARD L, RELAIX F, LAGHA M, ROCANCOURT D, DAUBAS P, BUCKINGHAM $M$ (2006). A novel genetic hierarchy functions during hypaxial myogenesis: Pax3 directly activates Myf5 in muscle progenitor cells in the limb. Genes Dev 20 2450-2464.

BELLAIRS R (1979). The mechanism of somite segmentation in the chick embryo. $J$ Embryol Exp Morphol 51: 227-243.

BERKES CA, TAPSCOTT SJ (2005). MyoD and the transcriptional control of myogenesis. Semin Cell Dev Biol 16: 585-595.

BISCHOFF R, HOLTZER H (1969). Mitosis and the processes of differentiation of myogenic cells in vitro. J Cell Biol 41: 188-200.

BLACKSHAW SE, WARNERAE (1976). Low resistance junctions between mesoderm cells during development of trunk muscles. J Physiol 255: 209-230.

BOUDJELIDAH, MUNTZL(1987). Multinucleation during myogenesis of the myotome of Xenopus laevis: a qualitative study. Development 101: 583-590.

BROHMANN H, JAGLA K, BIRCHMEIER C (2000). The role of Lbx1 in migration of muscle precursor cells. Development 127: 437-445.

BROWN DD (1964). RNA synthesis during amphibian development. J Exp Zool 157: $101-113$.

BRUSTIS JJ (1979). Ultrastructural aspects of the organization of somites and the early differentiation of myotomes in the embryo of the common toad Bufo bufo $\mathrm{L}$. Arch Biol (Liege) 90: 261-272.

BUCKINGHAM M (2003). How the community effect orchestrates muscle differentiation. BioEssays 25: 13-16.

BUCKINGHAM M (2006). Myogenic progenitor cells and skeletal myogenesis in vertebrates. Curr Opin Genet Dev 16: 525-532.

CHANOINE C, HARDY S (2003). Xenopus muscle development: from primary to secondary myogenesis. Dev Dyn 226: 12-23.

CHEEK DB, HOLTAB, HILL DE, TALBERT JL (1971). Skeletal Muscle Cell Mass and Growth: The Concept of the Deoxyribonucleic Acid Unit. Pediatr Res 5: 312-328.

CHRIST B, BRAND-SABERI B (2002). Limb muscle development. Int J Dev Biol 46: 905-914.

COOKE J, ZEEMAN EC (1976). A clock and wavefront model for control of the number of repeated structures during animal morphogenesis. J Theor Biol 58: 455-476.

COOPER WG, KONIGSBERG IR (1961). Dynamics of myogenesis in vitro. Anat 
Rec 140: 195-205.

DACZEWSKA M (2006). Comparative analysis of myotomal muscle differentiation in vertebrates with special reference to the role of mesenchymal cells. Zool Pol 51: 5-54.

DACZEWSKA M (2001). Mechanism of multinucleate myotomal muscle fibre formation in Hymenochirus boettgeri (Anura, Pipidae). Zoomorphology 121: 27-36.

DACZEWSKA M, KIEŁBÓWNA L (2000). Myotomal myogenesis in Triturus vulgaris L. (Urodela) with special reference to the role of mesenchymal cells. Folia Biol (Kraków) 48: 37-42.

DACZEWSKAM, PAŁUCKAM (1999). Development of primary and secondary muscle fibres in the myotomes of Rana Lessonae (Anura: Ranidae). Zool Pol 44: 59-69.

DACZEWSKA M, SACZKO J (2005). Myotomal myogenesis of axial muscle in the sturgeon Acipenser baeri (Chondrostei, Acipenseriformes). Folia Biol (Kraków) 53: 29-38.

DALE KJ, POURQUIÉ O (2000). A clock-work somite. BioEssays 22: 72-83.

DEVOTO SH, MELANÇON E, EISEN JS, WESTERFIELD M (1996). Identification of separate slow and fast muscle precursor cells in vivo, prior to somite formation. Development 122: 3371-3380.

FLOOD PR, KRYVIH, TOTLAND GK (1977). Onto-phylogenetic aspects of muscle fibre types in the segmental trunk muscle of lower chordates. Folia Morphol 25: 64-67.

FENG X, ADIARTE EG, DEVOTO SH (2006). Hedgehog acts directly on the zebrafish dermomyotome to promote myogenic differentiation. Dev Biol 300: 736-746.

GRIMALDIA, TETTAMANTIG, MARTINBL, GAFFIELDW, POWNALLME, HUGHES SM (2004). Hedgehog regulation of superficial slow muscle fibres in Xenopus and the evolution of tetrapod trunk myogenesis. Development 131: 3249-3262.

GURDON JB, TILLER E, ROBERTS J, KATO K (1993). A community effect in muscle development. Curr Biol 3: 1-11.

HAMILTON L (1969). The formation of somites in Xenopus. J Embryol Exp Morphol 22: 253-264.

HANOCQ-QUERTIER J, BALTUS E, FICQ A, BRACHET J (1968). Studies on the DNA of Xenopus laevis oocytes. J Embryol Exp Morphol 19: 273-282.

HARVEY RP (1992). MyoD protein expression in Xenopus embryos closely follows a mesoderm induction-dependent amplification of MyoD transcription and is synchronous across the future somite axis. Mech Dev 37: 141-149.

HOLT CE, LEMAIRE P, GURDON JB (1994). Cadherin-mediated cell interactions are necessary for the activation of MyoD in Xenopus mesoderm. Proc Natl Acad Sci USA 91: 10844-10848.

HOLTZER H (1970). Cell differentiation: myogenesis (Schjeide OA and de Vellis J, eds.) Van Nostrand, New York.

HOPWOOD ND, PLUCK A, GURDON JB (1989). MyoD expression in the forming somites is an early response to mesoderm induction in Xenopus embryos. EMBO J 8: 3409-3417.

HOPWOOD ND, PLUCK A, GURDON JB, DILWORTH SM (1992). Expression of XMyoD protein in early Xenopus laevis embryos. Development 114: 31-38.

JAGLA K, DOLLÉ P, MATTEI MG, JAGLA T, SCHUHBAUR B, DRETZEN G, BELLARD F, BELLARD M (1995). Mouse Lbx1 and human LBX1 define a novel mammalian homeobox gene family related to the Drosophila lady bird genes. Mech Dev 53: 345-356.

JEN WC, GAWANTKAV, FOLLET N, NIEHRS C, KINTNER C (1999). Periodic repression of Notch pathway genes governs the segmentation of Xenopus embryos. Genes Dev 13: 1486-1499.

JOSEPH EM, CASSETTA LA (1999). Mespo: A novel basic helix-loop-helix gene expressed in the presomitic mesoderm and posterior tailbud of Xenopus embryos. Mech Dev 82: 191-194.

JOUVEC, PALMEIRIM I, HENRIQUE D, BECKERS J, GOSSLERA, ISH-HOROWICZ D, POURQUIÉ O (2000). Notch signalling is required for cyclic expression of the hairy-like gene HES1 in the presomitic mesoderm. Development 127: 1421-1429.

KARASAKI S (1963). Studies on amphibian yolk. J Ultrastruct Res 9: 225-247.

KELLER R (2000). The origin and morphogenesis of amphibian somites. Curr Top Dev Biol 47: 183-246.

KELLEY RO, NAKAI GS, GUGANIG ME (1971). A biochemical and ultrastructura study of RNA in yolk platelets of Xenopus gastrulae. J Embryol Exp Morphol 26: 181-193. 48.

KIEŁBÓWNA L (1966). Cytological and cytophotometrical studies on myogenesis in
Xenopus laevis Daudin. Zool Pol 11: 247-255.

KIEŁBÓWNA L (1973). Cytophotometric investigations of the nuclear RNA and the analysis of the nucleoli in myogenesis of Xenopus laevis Daudin. Zool Pol 23 159-173.

KIEŁBÓWNA L (1993). Origin and development of musculus rectus abdominis in Bombina Bombina (Anura). Zool Pol 38: 39-51.

KIEŁBÓWNA L (1987). The development of primary and secoundary muscle fibres in myotomes of Pelobates Fuscus (Anura, Amphibia). Zool Pol 34: 169-184.

KIEŁBÓWNA L (1981). The formation of somites and early myotomal myogenesis in Xenopus laevis, Bombina variegata and Pelobates fuscus. J Embryol Exp Morphol 64: 295-304.

KIEŁBÓWNA L (1980). Two different types of myogenesis in Xenopus laevis Dudain Zool Pol 27: 377-395.

KIEŁBÓWNA L (1975). Utilization of yolk platelets and lipid bodies during the myogenesis of Xenopus laevis (Daudin). Cell Tissue Res 159: 279-86.

KIEŁBÓWNA L, DACZEWSKA M (2005). The origin of syncytial muscle fibres in the myotomes of Xenopus laevis--a revision. Folia Biol (Kraków) 53: 39-44.

KIEŁBÓWNAL, KOŚCIELSKIB (1979). Myotomal Myogenesis in Bombina variegate. L. Wilhelm Roux's Arch 185: 295-303.

KIEŁBÓWNA L, MIGOCKA-PATRZAŁEK M (2014). Molecular factors in myoblasts fusion. Postepy Biol Komorki 41: 559-581.

KORDYLEWSKI L, FABER J, GÖRLICH A, KILARSKI W (1989). Histochemistry and isomyosins of tail musculature in Xenopus. J Muscle Res Cell Motil 10: 290-296.

KRAUSS RS (2010). Regulation of promyogenic signal transduction by cell-cell contact and adhesion. Exp Cell Res 316: 3042-3049.

LIPTON BH, KONIGSBERG IR (1972). A fine-structural analysis of the fusion of myogenic cells. J Cell Biol 53: 348-364.

MALACINSKI G, NEFFA, RADICE G, CHUNG H (1989). Amphibian somite development: contrasts of morphogenetic and molecular differentiation patterns between the labolatory arkchetype species Xenopus (anuran) and axolotl (urodele). Zool Sci 6: 1-14.

MARTIN BL, HARLAND RM (2006). A novel role for lbx1 in Xenopus hypaxial myogenesis. Development 133: 195-208.

MARTIN BL, HARLAND RM (2001). Hypaxial muscle migration during primary myogenesis in Xenopus laevis. Dev Biol 239: 270-280.

MUNTZ L (1975). Myogenesis in the trunk and leg during development of the tadpole of Xenopus laevis (Daudin 1802). J Embryol Exp Morphol 33: 757-74.

NICOLAS N, GALLIEN CL, CHANOINE C (1998). Expression of myogenic regulatory factors during muscle development of Xenopus: myogenin mRNA accumulation is limited strictly to secondary myogenesis. Dev Dyn 213: 309-21.

NISHIDAH, SAWADAK (2001). Macho-1 encodes a localized mRNA in ascidian eggs that specifies muscle fate during embryogenesis. Nature 409(6821):724-729.

NISHIKAWA A, HAYASHI H (1994). Isoform transition of contractile proteins related to muscle remodeling with an axial gradient during metamorphosis in Xenopus laevis. Dev Biol 165: 86-94.

OKAZAKI K, HOLTZER H (1966). Myogenesis: fusion, myosin synthesis, and the mitotic cycle. Proc Natl Acad Sci USA 56: 1484-1490.

OLSON EN (1990). MyoD family: a paradigm for development? Genes Dev 4: 1454-1461.

OLSON EN, KLEIN WH (1994). bHLH factors in muscle development: dead lines and commitments, what to leave in and what to leave out. Genes Dev 8: 1-8.

ORLOV VS, SAMOSUDOVA N V, SHUNGSKAIA VE (1989). A possible physicochemical mechanism of cell fusion in skeletal muscle myogenesis by means of intercellular pinocytosis. Biofizika 34: 665-670.

PALMEIRIM I, HENRIQUE D, ISH-HOROWICZ D, POURQUIÉ O (1997). Avian hairy gene expression identifies a molecular clock linked to vertebrate segmentation and somitogenesis. Cell 91: 639-648.

RADICE GP, MALACINSKI GM (1989). Expression of myosin heavy chain transcripts during Xenopus laevis development. Int J Dev Biol 133: 562-568.

ROVAINEN CM (1979). Neurobiology of lampreys. Physiol Rev 59: 1007-1077.

ROMEK M (1998). Spatial distribution of yolk platelets and fat droplets in oocytes and cleaving embryos of the common frog (Rana temporaria) and toad (Bufo bufo). Folia Histochem Cytobiol 36: 87-96. 
SABO MC, NATH K, ELINSON RP (2009). Lbx1 expression and frog limb development. Dev Genes Evol 219: 609-612.

SAMESHIMA M, SHIOKAWA K (1969). Effects of 6-azauridine on the embryonic cells of Xenopus laevis, with special reference to nucleolar ultrastructure. J Exp Zool 170: 333-340.

SAWADA A, FRITZ A, JIANG YJ, YAMAMOTO A, YAMASU K, KUROIWA A, SAGA Y, TAKEDA H (2000). Zebrafish Mesp family genes, mesp-a and mesp-b are segmentally expressed in the presomitic mesoderm, and Mesp-b confers the anterior identity to the developing somites. Development 127: 1691-1702.

SCAALM, WIEGREFFE C (2006). Somite compartments in anamniotes. Anat Embryol (Berl) 211 (Suppl.) 1: 9-19.

SELMAN GG, PAWSEY GJ (1965). The utilization of yolk platelets by tissues of Xenopus embryos studied by a safranin staining method. J Embryol Exp Morphol 14: 191-212.

SPARROWDB, JEN WC, KOTECHAS, TOWERSN, KINTNERC, MOHUNTJ (1998). Thylacine 1 is expressed segmentally within the paraxial mesoderm of the Xenopus embryo and interacts with the Notch pathway. Development 125: 2041-2051.

STANDLEY HJ, ZORN AM, GURDON JB (2001). eFGF and its mode of action in the community effect during Xenopus myogenesis. Development 128: 1347-1357.

STICKNEY HL, BARRESI MJF, DEVOTO SH (2000). Somite development in Zebrafish. Dev Dyn 219: 287-303.
STOCKDALE FE, HOLTZER H (1961). DNA synthesis and myogenesis. Exp Cell Res 24: 508-520.

STOCKDALE FE, O'NEILL MC (1972). Deoxyribonucleic acid synthesis, mitosis, and skeletal muscle differentiation. In Vitro 8: 212-227.

STREHLER BL, KONIGSBERG IR, KELLEY JE (1963). Ploidy of myotube nuclei developing in vitro as determined with a recording double beam micro-spectrophotometer. Exp Cell Res 32: 232-241.

WEINTRAUB H, DAVIS R, TAPSCOTT S, THAYER M, KRAUSE M, BENEZRA R, BLACKWELL TK, TURNER D, RUPP R, HOLLENBERG S (1991). The myoD gene family: nodal point during specification of the muscle cell lineage. Science (80) 251: 761-766.

WILSON PA, OSTER G, KELLER R (1989). Cell rearrangement and segmentation in Xenopus: direct observation of cultured explants. Development 105: 155-166.

YOUN BW, MALACINSKI GM (1981a). Comparative analysis of amphibian somite morphogenesis: cell rearrangement patterns during rosette formation and myoblast fusion. J Embryol Exp Morphol 66: 1-26.

YOUN BW, MALACINSKI GM (1981b). Somitogenesis in the amphibian Xenopus laevis: scanning electron microscopic analysis of intrasomitic cellular arrangements during somite rotation. J Embryol Exp Morphol 64: 23-43.

YUAN J, ZHANG S, LIU Z, LUAN Z, HU G (2003). Cloning and phylogenetic analysis of an amphioxus myogenic bHLH gene AmphiMDF. Biochem Biophys Res Commun. 301: 960-967. 


\section{Further Related Reading, published previously in the Int. J. Dev. Biol.}

The Australian lungfish (Neoceratodus forsteri) - fish or amphibian pattern of muscle development? Agata Kacperczyk and Malgorzata Daczewska Int. J. Dev. Biol. (2008) 52: 279-286

http://dx.doi.org/10.1387/ijdb.072323ak

Distinctive expression of Myf5 in relation to differentiation and plasticity of newt muscle cells

Yutaka Imokawa, Phillip B. Gates, Young-Tae Chang, Hans-Georg Simon and Jeremy P. Brockes Int. J. Dev. Biol. (2004) 48: 285-291

http://dx.doi.org/10.1387/ijdb.031787yi

The community effect in Xenopus myogenesis is promoted by dorsalizing factors G Carnac and J B Gurdon

Int. J. Dev. Biol. (1997) 41: 521-524

http://dx.doi.org/10.1387/ijdb.9240569

Developmental histories in amphibian myogenesis

G P Radice, A W Neff, Y H Shim, J J Brustis and G M Malacinski

Int. J. Dev. Biol. (1989) 33: 325-343

http://dx.doi.org/10.1387/ijdb.2702121

Somitogenesis in the marsupial frog Gastrotheca riobambae

D Gatherer and $E$ M del Pino

Int. J. Dev. Biol. (1992) 36: 283-291

http://dx.doi.org/10.1387/ijdb.1525016

5 yr ISI Impact Factor $(2013)=2.879$
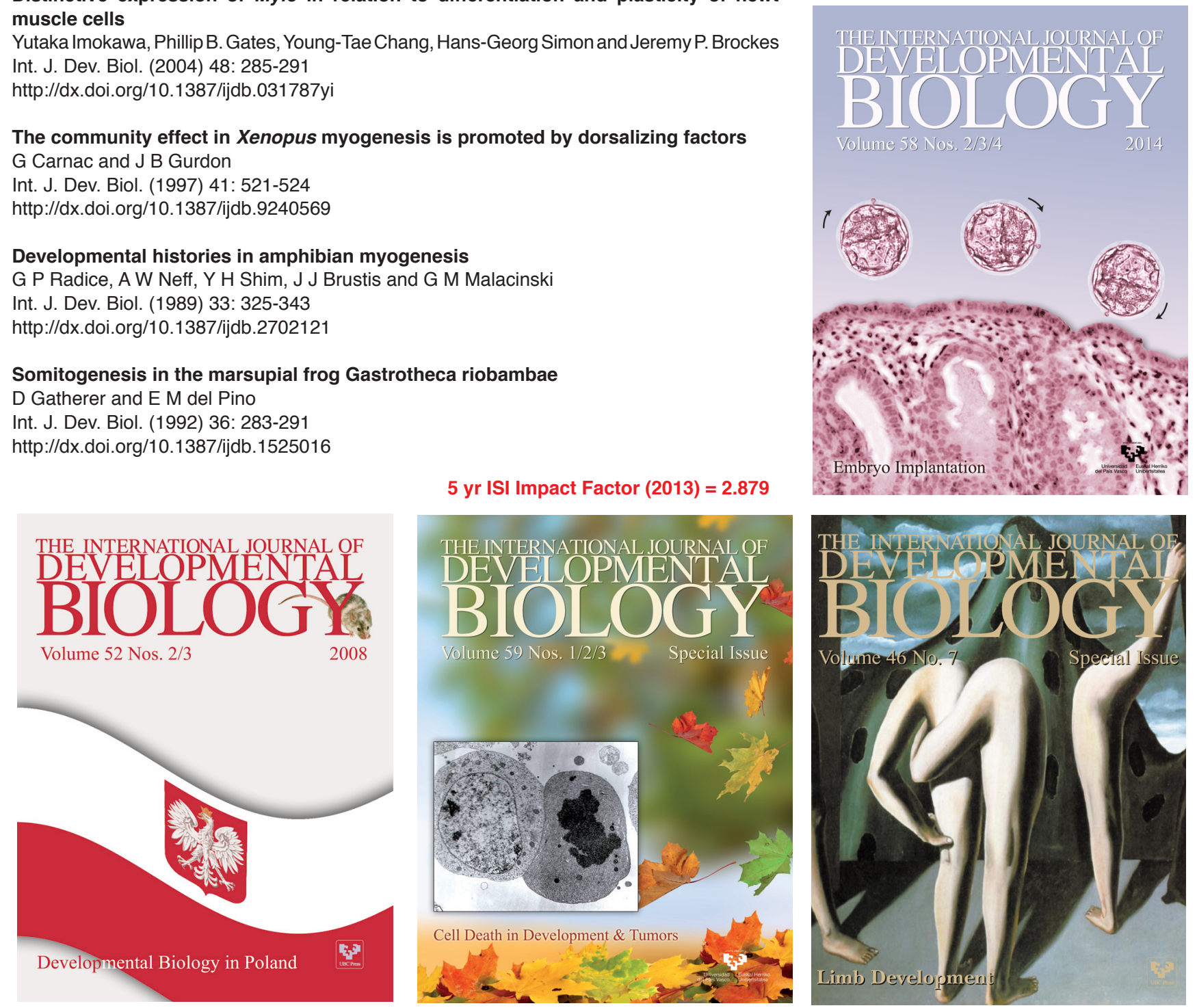